\title{
BMJ Open A multicentre, randomised controlled, non-inferiority trial, comparing high flow therapy with nasal continuous positive airway pressure as primary support for preterm infants with respiratory distress (the HIPSTER trial): study protocol
}

\author{
Calum T Roberts, ${ }^{1,2}$ Louise S Owen, ${ }^{1,2,3}$ Brett J Manley, ${ }^{1,2}$ Susan M Donath, ${ }^{3,4}$ \\ Peter G Davis ${ }^{1,2,3}$
}

To cite: Roberts CT, Owen LS, Manley BJ, et al. A multicentre, randomised controlled, non-inferiority trial, comparing high flow therapy with nasal continuous positive airway pressure as primary support for preterm infants with respiratory distress (the HIPSTER trial): study protocol. BMJ Open 2015;5 e008483. doi:10.1136/ bmjopen-2015-008483

- Prepublication history for this paper is available online To view these files please visit the journal online (http://dx.doi.org/10.1136/ bmjopen-2015-008483).

Received 14 April 2015 Accepted 13 May 2015

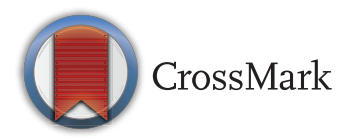

For numbered affiliations see end of article.

Correspondence to Dr Calum T Roberts; calum. roberts@thewomens.org.au

\section{ABSTRACT}

Introduction: High flow (HF) therapy is an increasingly popular mode of non-invasive respiratory support for preterm infants. While there is now evidence to support the use of HF to reduce extubation failure, there have been no appropriately designed and powered studies to assess the use of HF as primary respiratory support soon after birth. Our hypothesis is that $\mathrm{HF}$ is non-inferior to the standard treatmentnasal continuous positive airway pressure (NCPAP)— as primary respiratory support for preterm infants. Methods and analysis: The HIPSTER trial is an unblinded, international, multicentre, randomised, noninferiority trial. Eligible infants are preterm infants of $28-36^{+6}$ weeks' gestational age (GA) who require primary non-invasive respiratory support for respiratory distress in the first $24 \mathrm{~h}$ of life. Infants are randomised to treatment with either HF or NCPAP. The primary outcome is treatment failure within $72 \mathrm{~h}$ after randomisation, as determined by objective oxygenation, blood gas, and apnoea criteria, or the need for urgent intubation and mechanical ventilation. Secondary outcomes include the incidence of intubation, pneumothorax, bronchopulmonary dysplasia, nasal trauma, costs associated with hospital care and parental stress. With a specified noninferiority margin of $10 \%$, using a two-sided $95 \% \mathrm{Cl}$ and $90 \%$ power, the study requires 375 infants per group (total 750 infants).

Ethics and dissemination: Ethical approval has been granted by the relevant human research ethics committees at The Royal Women's Hospital (13/12), The Royal Children's Hospital (33144A), The Mercy Hospital for Women (R13/34), and the South-Eastern Norway Regional Health Authority (2013/1657). The trial is currently recruiting at 9 centres in Australia and Norway. The trial results will be published in peerreviewed international journals, and presented at national and international conferences.

\section{Strengths and limitations of this study}

- This is the first study that is appropriately designed and powered to assess the efficacy of high flow therapy as primary respiratory support for preterm infants.

- The use of a non-inferiority design is appropriate given the advantages of high flow over nasal continuous positive airway pressure. A narrow non-inferiority margin $(10 \%)$ has been chosen to ensure the study results will be convincing to clinicians.

- Blinding of the allocated respiratory support modes is not possible, but objective criteria are specified for the primary outcome of treatment failure.

- Some infants in the high flow group will have initially received a brief period of nasal continuous positive airway pressure prior to randomisation.

Trial registration number: Australian New Zealand Clinical Trials Registry ID: ACTRN12613000303741.

\section{INTRODUCTION}

\section{Background}

Preterm birth is the leading cause of newborn death worldwide. Every year, 15 million infants are born preterm and $>1$ million die from complications. ${ }^{1}$ Respiratory distress syndrome (RDS) is one such complication occurring in $44 \%$ of very low birthweight infants $(<1500 \mathrm{~g}) ;^{2}$ therefore, identifying the optimal method for providing 
breathing support is crucial for this group. Ventilation via an endotracheal tube (ETT) has improved preterm survival, but has increased rates of lung damage. ${ }^{3}$ As a result, 'non-invasive' techniques (without an ETT) have been developed to minimise lung damage. Nasal continuous positive airway pressure (NCPAP) is an effective mode of support for newborn infants with respiratory distress. It reduces extubation failure in previously ventilated infants, ${ }^{4}$ and is an effective alternative to intubation and mechanical ventilation at birth for preterm infants with RDS. ${ }^{5}$ Published randomised trials have reported successful use of NCPAP without intubation and mechanical ventilation in $48-54 \%$ of infants born at 25-30 weeks. ${ }^{6} 7$ NCPAP also significantly reduces the need for transfer of infants $>30$ weeks' gestation with respiratory distress, born in non-tertiary neonatal units. ${ }^{8}$

Unfortunately, NCPAP has significant limitations; the need for the prongs to completely fill the nostrils can result in damage to the nasal mucosa and septum. ${ }^{9}$ Excessive leak around the prongs and through the mouth can lead to inadequate support, whereas excessive pressure may result in pneumothoraces; ${ }^{6}$ both of these may require intubation and ventilation. Pressurised gas can cause abdominal distension, ${ }^{10}$ and the bulky fixation devices obscure the infant's face. Both of these problems interfere with feeding and positioning. These challenges are driving the search for alternative treatments.

In recent years, high flow (HF) therapy has become popular and is used in many neonatal intensive care units (NICUs) in the USA ${ }^{11}$ as well as in NICUs and non-tertiary neonatal units within the UK, Australia and New Zealand. ${ }^{12-14}$

HF refers to heated, humidified, blended oxygen delivered into the nose via loose fitting short binasal prongs, at a flow of at least $1 \mathrm{~L} / \mathrm{min}^{15}{ }^{15}$

While the use of commercially available HF systems has been adopted by many NICUs, there is relatively little evidence to support its efficacy as respiratory support in the neonatal population, particularly when used as the primary mode of respiratory support. The popularity of HF seems to be due to other perceived advantages, for example, that the cannulae are easier to apply than NCPAP prongs, may be more comfortable for infants, may be associated with less nasal trauma and may enable easier access to babies' faces, thus allowing for greater opportunities for feeding and parental bonding. ${ }^{17}$ If $\mathrm{HF}$ therapy was demonstrated to be as effective as NCPAP, these other factors might lead to it being preferred in clinical practice.

\section{Evidence of clinical efficacy of HF}

Pooled analysis of nine randomised controlled trials (RCTs) has demonstrated that NCPAP prevents extubation failure, in comparison to ambient oxygen alone. ${ }^{4}$ A 2011 Cochrane review of four randomised studies comparing NCPAP with HF in 177 infants found the trials unsuitable for meta-analysis due to methodological differences, and concluded there was insufficient evidence to support the use of $\mathrm{HF}$ as post-extubation respiratory support in preterm infants. ${ }^{15}$

In 2013, three RCTs were published, comparing NCPAP with HF as post-extubation respiratory support. Studies by Collins ${ }^{18}$ and Manley ${ }^{19}$ included 435 very preterm infants ( $<32$ weeks' gestation) randomised at extubation, and both trials demonstrated no significant difference in extubation failure within 7 days between the NCPAP and HF groups. Yoder ${ }^{20}$ conducted a trial including 432 infants of $\geq 28$ weeks' gestation (of whom 291 were randomised at extubation). There was no significant difference between the study groups for the primary outcome of intubation/reintubation within the first $72 \mathrm{~h}$ of treatment. These three trials suggest that $\mathrm{HF}$ is a viable alternative to NCPAP as post-extubation support.

While NCPAP is well established as a primary respiratory support mode for preterm infants, there is little evidence for $\mathrm{HF}$ in this setting. A retrospective review of infants initially treated with $\mathrm{HF}$, compared with an earlier cohort managed with NCPAP, found fewer early intubations in the HF cohort. ${ }^{21}$ The largest of the aforementioned RCTs included a subgroup of 141 infants who had not been previously intubated and ventilated. Although these infants were not analysed separately, the data are encouraging and show that HF may be a useful therapy for early respiratory distress. A small pilot RCT including 38 preterm infants managed with either early HF or nasal intermittent positive pressure ventilation demonstrated no difference in treatment failure. However, there are no appropriately designed and powered RCTs comparing HF with NCPAP as primary treatment for early RDS in preterm infants.

\section{Potential advantages and safety of HF}

Concerns that airway pressures generated by HF could be very high have been allayed by an accumulation of data demonstrating that HF generates airway pressures at, or below, those resulting from NCPAP, especially when a leak is maintained around the prongs. ${ }^{22-25}$

In late 2005, one of the commercially available HF devices was associated with bacterial infection. ${ }^{26} \mathrm{~A}$ worldwide brand recall took place before the device was reintroduced in early 2007, without further reported problems.

Preterm infants with RDS are at risk of pneumothorax, a recognised complication of NCPAP therapy. ${ }^{5} 6$ Pneumothorax is also a potential risk in HF therapy. However, reports of pneumothoraces in preterm infants treated with $\mathrm{HF}$ are rare; only two cases were reported from the 431 infants randomised to receive HF in recent RCTs, ${ }^{18-20}$ compared with $10 / 436$ infants randomised to receive NCPAP. However, most infants in these studies had previously been intubated and received surfactant replacement, therefore pneumothorax rates would be expected to be low. Preterm infants treated with primary NCPAP from birth, who have not received surfactant, 
have much higher pneumothorax rates of up to $9 \%{ }^{6} 8$ the risk of pneumothorax during primary HF support is unknown.

Three RCTs have convincingly demonstrated that HF results in less nasal trauma than NCPAP. ${ }^{18-20}$ Further studies have shown HF is preferred by parent ${ }^{27}$ and by nursing staff. ${ }^{28}$ Other perceived advantages of HF, such as greater infant comfort and better establishment of feeding, remain unproven.

\section{Rationale and aim}

Neonatal HF use, including use as primary support, is rapidly increasing around the world. It is crucial that HF therapy is applied without causing harm by making appropriate assessment of its use before it becomes widely accepted in the neonatal practice. If HF does provide comparable support to NCPAP for preterm infants with early respiratory distress, then it is likely that it will be widely adopted in preference to NCPAP in NICUs as it is easier to use, more comfortable for infants ${ }^{29}$ reduces nasal trauma, and is preferred by clinicians and parents. ${ }^{14} 1727$

The aim of this study is to assess whether HF is noninferior to NCPAP in preventing treatment failure, when used as primary respiratory support for preterm infants.

\section{METHODS}

\section{Study design}

HIPSTER is an international, multicentre, randomised, non-inferiority trial, conducted in preterm infants $\geq 28$ weeks' gestational age (GA) requiring primary noninvasive respiratory support for respiratory distress in the first $24 \mathrm{~h}$ of life.

\section{Blinding}

The intervention in this study cannot be blinded. To limit bias, predefined, objective criteria for the primary outcome of treatment failure are specified so as to provide clear directions to clinicians for the decision to escalate respiratory support.

\section{Primary outcome}

The primary outcome is treatment failure within $72 \mathrm{~h}$ after randomisation. Treatment failure is reached once an infant is receiving maximal therapy for their allocated treatment (NCPAP $8 \mathrm{~cm} \mathrm{H}_{2} \mathrm{O}$ or $\mathrm{HF} 8 \mathrm{~L} / \mathrm{min}$ ), plus at least one of:

1. Sustained increase in oxygen requirement above $\geq 40 \%$ to maintain oxygen saturation in the target range for that centre;

2. Frequent apnoea: six or more apnoeas requiring intervention in a 6 -h period, or two or more apnoeas requiring facemask positive pressure ventilation in a 24-h period;

3. Respiratory acidosis: blood $\mathrm{pH} \leq 7.20$ and carbon dioxide $>60 \mathrm{~mm} \mathrm{Hg}$ of mercury on capillary/arterial blood, taken at least an hour after commencing the assigned treatment.

Treatment failure will also be adjudged to have occurred in any infant requiring urgent intubation and mechanical ventilation, as determined by the treating clinician.

\section{Secondary outcomes}

1. Reason(s) for 'treatment failure'

2. Intubation rate in first $72 \mathrm{~h}$, and at any time

3. Incidence of radiologically confirmed pneumothorax or other air leak

4. Incidence of significant nasal trauma (as measured using a validated nasal trauma scoring chart)

5. Incidence of bronchopulmonary dysplasia (supplemental oxygen requirement and/or need for respiratory support at 36 weeks' postmenstrual age)

6. Use of postnatal steroids for the treatment of lung disease

7. Discharged home with supplemental oxygen

8. Duration of admission, days of each respiratory support mode, death before discharge

9. Incidence of important neonatal morbidities including: late-onset sepsis, patent ductus arteriosus, necrotising enterocolitis, intestinal perforation, severe intraventricular haemorrhage, and treated retinopathy of prematurity

10. Days to reach full enteral feeds and full suck feeds, method of feeding at discharge, and weight gain until discharge

11. Economic analyses (overseen by a trial health economist)

12. Parental stress and perception of infant's treatment, as measured by a validated questionnaire: ('Parental Stress Scale: Neonatal Intensive Care Unit', PSS: $\mathrm{NICU}^{30}$ ).

\section{Setting}

The trial will be conducted in nine tertiary level NICUs ( 4 centres in Australia and 5 centres in Norway). All centres routinely care for preterm infants with respiratory distress, and use NCPAP as their standard mode of primary respiratory support.

\section{Eligibility criteria}

Inclusion: Infants will be included if

1. They are born at $28-36^{+6}$ weeks GA, AND

2. They are admitted to a participating NICU (inborn or outborn) when $<24 \mathrm{~h}$ old, AND

3. The decision has been made by the attending clinician, to commence or continue (from stabilisation at birth) non-invasive respiratory support (this does not include the provision of supplemental oxygen alone), AND

4. They have not previously been intubated or received surfactant, AND

5. At randomisation, the infant has received $<4 \mathrm{~h}$ of NCPAP support (respiratory support may need to 
start prior to consent being obtained and if so, this will be with NCPAP).

Exclusion: Infants will be excluded if

1. They immediately require intubation and ventilation (determined by attending clinician), OR

2. They already satisfy 'treatment failure' criteria, OR

3. They have a known major congenital anomaly or air leak (pneumothorax).

\section{Randomisation}

Prerandomisation stratification is by GA $(<32$ and $\geq 32$ weeks') and by study centre. Multiple births will be randomised individually. The randomisation sequence is computer generated with variable block sizes; assigned treatment is provided in consecutively numbered, sealed opaque envelopes.

\section{Clinical management}

Eligible infants will be randomised to treatment with either HF or NCPAP. Infants with birth weight $\leq 1250 \mathrm{~g}$ will receive caffeine for apnoea prevention ${ }^{31}$ at enrolment if not already given, to be continued at least during the primary outcome period. Apnoeic infants $>1250 \mathrm{~g}$ may receive caffeine as per the clinician's discretion. Infants in both groups will receive standard supportive care as per individual unit protocols, for example, blood tests, X-rays, antibiotics, intravenous fluid/nutrition and enteral feeds.

\section{Standard care: control group (NCPAP)}

1. NCPAP will be delivered using any NCPAP delivery device and short binasal prongs; pressure will start at 6-8 $\mathrm{cm} \mathrm{H}_{2} \mathrm{O}$ (clinician discretion). Pressure changes will be made with $1 \mathrm{~cm} \mathrm{H}_{2} \mathrm{O}$ increments/decrements in the range $5-8 \mathrm{~cm} \mathrm{H}_{2} \mathrm{O}$. Weaning will be reviewed at least daily with cessation considered once the infant is stable on NCPAP $5 \mathrm{~cm} \mathrm{H}_{2} \mathrm{O}$, in $<30 \%$ oxygen, for $>24 \mathrm{~h}$. Subsequently, unconditioned 'low flow' oxygen may be given to maintain oxygen saturation

2. Infants in the NCPAP group will not receive HF unless there is significant nasal trauma (defined as $\geq$ stage 2 on the study nasal trauma chart)

3. Infants who reach treatment failure criteria while receiving maximal NCPAP $\left(8 \mathrm{~cm} \mathrm{H}_{2} \mathrm{O}\right)$ within the primary outcome period $(72 \mathrm{~h}$ ) will be intubated and ventilated.

If non-invasive respiratory support is required later during admission (either postextubation or for later deterioration), NCPAP should be used, unless there is significant nasal trauma.

\section{Intervention group (HF)}

1. HF will be given using either Optiflow Junior (Fisher \& Paykel Healthcare, New Zealand) or Vapotherm (Vapotherm, Exeter, USA). Gas flow will start at 6$8 \mathrm{~L} / \mathrm{min}$ (clinician discretion), and flow changes will be made with $1 \mathrm{~L} / \mathrm{min}$ increments/decrements in the range $4-8 \mathrm{~L} / \mathrm{min}$. Weaning will be reviewed at least daily with cessation considered once the infant is stable on $4 \mathrm{~L} / \mathrm{min}$, in $<30 \%$ oxygen, for $>24 \mathrm{~h}$. Subsequently, unconditioned 'low flow' oxygen may be given to maintain oxygen saturation

2. Infants who reach treatment failure criteria while receiving maximal $\mathrm{HF}(8 \mathrm{~L} / \mathrm{min})$ within the primary outcome period $(72 \mathrm{~h})$ will receive NCPAP at $7-$ $8 \mathrm{~cm} \mathrm{H}_{2} \mathrm{O}$ (clinician discretion)

3. Infants who again reach treatment failure criteria while receiving maximal NCPAP $\left(8 \mathrm{~cm} \mathrm{H}_{2} \mathrm{O}\right)$, while still within the 72-h primary outcome period, will be intubated and ventilated

4. If further non-invasive respiratory support is required later during admission (eg, for clinical deterioration), infants should receive HF. However, if they have previously reached treatment failure criteria during HF, they may be treated with NCPAP at clinician discretion.

\section{Sample size calculation}

A review of preterm infants $>28$ weeks' GA receiving NCPAP as their initial mode of respiratory support at the participating Australian centres (unpublished data) showed that $17 \%$ of such infants were subsequently intubated and ventilated, within $72 \mathrm{~h}$ of starting treatment. We, therefore, chose an expected NCPAP 'treatment failure' rate of $17 \%$.

We have set the margin of non-inferiority for the trial at $10 \%$. That is, $\mathrm{HF}$ will be considered non-inferior to NCPAP if the risk difference for treatment failure and upper limit of its two-sided $95 \%$ CI is $<10 \%{ }^{32}$ (eg, if the NCPAP treatment failure rate is $17 \%$, both the risk difference and upper limit of its two-sided 95\% CI must be $<27 \%$ ). To demonstrate this with $90 \%$ power, we require a sample size of 375 infants per group, 750 infants in total. We chose this margin of non-inferiority with consideration to the following factors:

- HF is already a widely accepted mode of respiratory support in many tertiary and non-tertiary neonatal units;

- Infants in whom HF treatment fails will receive NCPAP, and we hypothesise that this will 'rescue' some of these infants from intubation;

- The primary outcome of this study is treatment failure, as opposed to an outcome like death or severe disability, when a lower margin of noninferiority would be necessary;

- This non-inferiority margin was thought to be appropriate, and was agreed on by all neonatologists in all the participating centres, and by parent representatives consulted during the trial design phase.

\section{Statistical analysis}

The incidence of the primary outcome will be compared using risk difference and two-sided 95\% CI. Planned subgroup analyses by GA strata will be performed for the primary outcome. Secondary outcomes will be compared using risk difference $(95 \% \mathrm{CI})$ and $\chi^{2}$ tests, or the 
appropriate parametric (t test) or non-parametric (Mann-Whitney U) tests. Statistical analyses will be by intention to treat, conforming to the Consort reporting guidelines.

Cost-effectiveness analysis will incorporate the costs of the device and of hospital care; a decision analysis will be constructed based on the primary outcome and associated hospital costs. Univariate and probabilistic sensitivity analyses will be conducted as a cost per additional treatment failure avoided for HF versus NCPAP.

\section{ETHICS AND DISSEMINATION}

\section{Research ethics approval}

The HIPSTER trial has received multisite ethical approval from the relevant governing bodies for all participating centres.

\section{Recruitment and consent}

Written parental consent is required for all infants participating in the trial. Consent will be sought in the antenatal period when possible, at all sites. When antenatal consent is in place, infants will be randomised as soon as possible after meeting eligibility criteria.

When antenatal consent has not been obtained, infants judged to require non-invasive respiratory support will receive standard treatment (NCPAP) until consent has been given. Families of infants meeting eligibility criteria will be approached at the earliest opportunity after birth, and before their infant has received 4 $h$ of NCPAP treatment.

Additionally, at the lead centre (The Royal Women's Hospital, Melbourne), the Human Research Ethics Committee (HREC) has approved a retrospective consent process. Eligible infants who have not been consented antenatally can be randomised as soon as they meet eligibility criteria. Their parents will then be approached for consent in the first few days after trial entry, at which point the parents may choose to give consent for their infant to remain in the trial, or remove them and opt for standard treatment.

The consent process, whether antenatal or postnatal, will include both a full verbal explanation of the trial and the use of the written patient information and consent form.

\section{Data collection and storage}

Outcome data, birth details and parental demographics will be collected from the infant's records and mother's medical records, and by parental interview. Data will be de-identified and entered into a paper case record form. Data will subsequently be entered into REDCap (Research Electronic Data Capture) ${ }^{33}$ - a secure, password-protected electronic database.

\section{Monitoring and safety}

A data safety monitoring committee (DSMC) comprising of two independent neonatologists and an independent statistician has been appointed. Set DSMC review points on the progress and safety of the trial are after the primary outcome is known for 250 and 500 infants. While no formal stopping rule will be used, the DSMC may recommend ceasing the trial if there is a statistically significant difference $(p<0.001)$ in primary outcome between the treatment groups overall or within prespecified GA subgroups, ${ }^{32}$ or in case of serious adverse events (identified as pneumothorax or other air leak from the lung while receiving the assigned treatment, and death before discharge). Cessation of the trial may also be recommended if there is equipment failure or recall, or if other evidence becomes available that would make continuing the trial unethical. All serious adverse events are to be reported to the lead centre's Human Research and Ethics Committee, and will be reviewed by the DSMC at the prespecified monitoring points. The first review point was reached in October 2014 and the DSMC recommended that the trial continue without modification.

\section{Dissemination of results}

Trial results will be published in peer-reviewed international journals, and presented at relevant national and international conferences. A plain language summary of the results will be sent to the parents of participants.

\section{Current status and study duration}

The trial began single-site recruitment in May 2013; it became multicenter in January 2014 and was extended to international sites in September 2014, and is currently recruiting in all nine participating centres. It is expected that recruitment will be completed in 2016 .

\section{Trial registration}

The HIPSTER trial is registered with the Australian

New Zealand Clinical Trials Registry (ID: ACTRN12613000303741).

\section{DISCUSSION}

HF therapy has been widely adopted in neonatal practice due to its desirable qualities such as ease of use, reduced nasal trauma, and parental and nursing preference. ${ }^{12}{ }^{13}$ However, it is of concern that HF is being used as primary respiratory support for preterm infants in the absence of good quality evidence of its efficacy in this setting. The HIPSTER trial is the first appropriately powered and designed trial to assess HF as primary support for preterm infants.

Non-inferiority trials are relatively uncommon in neonatal practice, but appropriate in this case due to the advantages associated with $\mathrm{HF}$, which would make it preferable to NCPAP provided that it is non-inferior in efficacy. The choice of non-inferiority margin is important in such a trial, and our margin of $10 \%$ was chosen in view of the fact that the primary outcome was 
treatment failure and not a more critical outcome, such as death, and that infants who have treatment failure on HF will be offered NCPAP, which may 'rescue' them from intubation and ventilation. This non-inferiority margin is half the size of that used in a previous postextubation trial of $\mathrm{HF}$ published by our group ${ }^{19}$ given that the expected rate of treatment failure in the population of The HIPSTER trial, a study of primary respiratory support, is lower, and therefore the criteria for non-inferiority should be stricter.

A potential limitation to this trial is that blinding is not possible. We have attempted to minimise this by setting objective treatment failure criteria, which were agreed on by all participating centres. Some infants randomised to $\mathrm{HF}$ will have received a brief period of NCPAP before randomisation, which conceivably could affect interpretation of the results. However, we have aimed to restrict the impact of this by making any infant who has received four or more hours of NCPAP ineligible for the trial and by the use of antenatal consent when possible, and a retrospective consent process at the lead centre. Acceptance of such a process requires the approval of both the HREC and the treating clinical team, and this may vary from site to site. We feel retrospective consent is appropriate in this trial given that $\mathrm{HF}$ has already been adopted into standard practice as a mode of primary respiratory support by some neonatologists ${ }^{12}$ and that along with the inclusion of 'rescue' NCPAP, the HREC adjudged that infants in the HF group were not exposed to additional risk in comparison to those treated with NCPAP.

The use of HF in neonatal practice is now well established, but good quality evidence is required to determine in which clinical settings this is appropriate. If this trial demonstrates that $\mathrm{HF}$ is non-inferior to NCPAP as primary support, then this practice is likely to be widely adopted around the world. However, if $\mathrm{HF}$ is inferior to NCPAP, then this study will ensure that preterm infants, who require non-invasive respiratory support, receive the optimal treatment.

\author{
Author affiliations \\ ${ }^{1}$ The Royal Women's Hospital, Melbourne, Australia \\ ${ }^{2}$ Department of Obstetrics and Gynaecology, The University of Melbourne, \\ Melbourne, Australia \\ ${ }^{3}$ Murdoch Children's Research Institute, Melbourne, Australia \\ ${ }^{4}$ Department of Paediatrics, The University of Melbourne, Melbourne, Australia
}

Acknowledgements The authors wish to thank the following for their contribution to the trial: HIPSTER investigators: TE Calisch, D Cartwright, C Collins, G Flagstad, D Fugelseth, DH Frøisland, M Grønn, C Klingenberg, A Leknessund, A Malhotra, B Nakstad, M Pritchard, A Ronnestad, AL Solevåg. Research nurses/assistants: B Argus, MI Austgulen, A Brett, U Grepperud, KG Gustavsen, EA Hansen, B Høium, A Jørstad, G Lack, E Ludvigsen, PJ Lyseggen, L McKeown, B Mills, CS Nygaard, C Wong, E Yeomans. Royal Women's Hospital Research Fellows: L McGrory, J O'Shea. Data Safety Monitoring Committee: H Liley (chair), K Lee, K Wheeler.

Contributors CTR conceived and designed the trial protocol, wrote the first draft and revised the manuscript for intellectual content. LSO, BJM and PGD conceived and designed the trial protocol and revised the manuscript for important intellectual content. SMD designed the protocol statistical analysis and revised the manuscript for important intellectual content. All the authors have read and approved the final manuscript, and are accountable for its accuracy.

Funding The HIPSTER trial is supported by a National Health and Medical Research Council (NHMRC, Australia) project grant (No. 1079089), a NHMRC Centre for Research Excellence grant (No. 1060733), and a grant from the Royal Brisbane and Women's Hospital (Australia) Foundation.

\section{Competing interests None declared.}

Ethics approval The trial is approved by: the Royal Women's Hospital Human Research Ethics Committee (Reference: 13/12), the Royal Children's Hospital Human Research Ethics Committee (Reference: 33144A), the Mercy Hospital for Women Human Research Ethics Committee (Reference: R13/34), and South-Eastern Norway Regional Health Authority Committee for Medical and Health Research (Reference: 2013/1657)

Provenance and peer review Not commissioned; peer reviewed for ethical and funding approval prior to submission.

Data sharing statement Further information on the study protocol may be requested from the corresponding author.

Open Access This is an Open Access article distributed in accordance with the Creative Commons Attribution Non Commercial (CC BY-NC 4.0) license, which permits others to distribute, remix, adapt, build upon this work noncommercially, and license their derivative works on different terms, provided the original work is properly cited and the use is non-commercial. See: http:// creativecommons.org/licenses/by-nc/4.0/

\section{REFERENCES}

1. Blencowe $\mathrm{H}$, Cousens $\mathrm{S}$, Oestergaard $\mathrm{MZ}$, et al. National, regional, and worldwide estimates of preterm birth rates in the year 2010 with time trends since 1990 for selected countries: a systematic analysis and implications. Lancet 2012;379:2162-72.

2. Fanaroff AA, Stoll BJ, Wright LL, et al. Trends in neonatal morbidity and mortality for very low birthweight infants. Am J Obstet Gynecol 2007;196:147 e1-8.

3. Chess PR, D'Angio CT, Pryhuber GS, et al. Pathogenesis of bronchopulmonary dysplasia. Semin Perinatol 2006:30:171-8.

4. Davis PG, Henderson-Smart DJ. Nasal continuous positive airways pressure immediately after extubation for preventing morbidity in preterm infants. Cochrane Database Syst Rev 2003(2):CD000143.

5. Finer NN, Carlo WA, Walsh MC, et al. Early CPAP versus surfactant in extremely preterm infants. N Engl J Med 2010;362:1970-9.

6. Morley CJ, Davis PG, Doyle LW, et al. Nasal CPAP or intubation at birth for very preterm infants. N Engl J Med 2008;358:700-8.

7. Dunn MS, Kaempf J, de Klerk A, et al. Randomized trial comparing 3 approaches to the initial respiratory management of preterm neonates. Pediatrics 2011;128:e1069-76.

8. Buckmaster AG, Arnolda G, Wright IM, et al. Continuous positive airway pressure therapy for infants with respiratory distress in non tertiary care centers: a randomized, controlled trial. Pediatrics 2007:120:509-18.

9. Robertson NJ, McCarthy LS, Hamilton PA, et al. Nasal deformities resulting from flow driver continuous positive airway pressure. Archives of disease in childhood Fetal and neonatal edition 1996;75: F209-12.

10. Jaile JC, Levin T, Wung JT, et al. Benign gaseous distension of the bowel in premature infants treated with nasal continuous airway pressure: a study of contributing factors. AJR Am J Roentgenol 1992:158:125-7.

11. Hochwald $\mathrm{O}$, Osiovich $\mathrm{H}$. High flow nasal cannulae in neonatal intensive care units: is clinical practice consistent with the evidence. Paper presented at: PAS Annual Meeting; 1-4 May 2010; Vancouver, British Columbia, 2010.

12. Hough JL, Shearman $A D$, Jardine $L A$, et al. Humidified high flow nasal cannulae: current practice in Australasian nurseries, a survey. $J$ Paediatr Child Health 2012;48:106-13.

13. Nath $\mathrm{P}$, Ponnusamy $\mathrm{V}$, Willis $\mathrm{K}$, et al. Current practices of high and low flow oxygen therapy and humidification in UK neonatal units. Pediatr Int 2010;52:893-4.

14. Manley BJ, Owen L, Doyle LW, et al. High-flow nasal cannulae and nasal continuous positive airway pressure use in non-tertiary special care nurseries in Australia and New Zealand. $J$ Paediatr Child Health 2012;48:16-21. 
15. Wilkinson D, Andersen C, O'Donnell CP, et al. High flow nasal cannula for respiratory support in preterm infants. Cochrane Database Syst Rev 2011(5):CD006405.

16. Waugh JB, Granger WM. An evaluation of 2 new devices for nasal high-flow gas therapy. Respir Care 2004;49:902-6.

17. Manley BJ, Dold SK, Davis PG, et al. High-flow nasal cannulae for respiratory support of preterm infants: a review of the evidence. Neonatology 2012;102:300-8

18. Collins CL, Holberton JR, Barfield C, et al. A randomized controlled trial to compare heated humidified high-flow nasal cannulae with nasal continuous positive airway pressure postextubation in premature infants. J Pediatr 2013;162:949-54 e1.

19. Manley BJ, Owen LS, Doyle LW, et al. High-flow nasal cannulae in very preterm infants after extubation. $N$ Engl $J$ Med 2013;369:1425-33.

20. Yoder BA, Stoddard RA, Li M, et al. Heated, humidified high-flow nasal cannula versus nasal CPAP for respiratory support in neonates. Pediatrics 2013;131:e1482-90.

21. Shoemaker MT, Pierce MR, Yoder BA, et al. High flow nasal cannula versus nasal CPAP for neonatal respiratory disease: a retrospective study. J Perinatol 2007;27:85-91.

22. Lampland AL, Plumm B, Meyers PA, et al. Observational study of humidified high-flow nasal cannula compared with nasal continuous positive airway pressure. J Pediatr 2009;154:177-82.

23. Sivieri EM, Gerdes JS, Abbasi S. Effect of HFNC flow rate, cannula size, and nares diameter on generated airway pressures: an in vitro study. Pediatr Pulmonol 2012.

24. Spence KL, Murphy D, Kilian C, et al. High-flow nasal cannula as a device to provide continuous positive airway pressure in infants. J Perinatol 2007;27:772-5.
25. Wilkinson DJ, Andersen CC, Smith K, et al. Pharyngeal pressure with high-flow nasal cannulae in premature infants. J Perinatol 2008;28:42-7.

26. Jhung MA, Sunenshine RH, Noble-Wang J, et al. A national outbreak of Ralstonia mannitolilytica associated with use of a contaminated oxygen-delivery device among pediatric patients. Pediatrics 2007:119:1061-8.

27. Klingenberg C, Pettersen M, Hansen EA, et al. Patient comfort during treatment with heated humidified high flow nasal cannulae versus nasal continuous positive airway pressure: a randomised cross-over trial. Arch Dis Child Fetal Neonatal Ed 2014:99:F134-7.

28. Roberts CT, Manley BJ, Dawson JA, et al. Nursing perceptions of high-flow nasal cannulae treatment for very preterm infants. $J$ Paediatr Child Health 2014:50:806-10.

29. Osman M, Elsharkawy A, Abdel-Hady H. Assessment of pain during application of nasal-continuous positive airway pressure and heated humidified high-flow nasal cannulae in preterm infants. J Perinatol 2015;35:263-7.

30. Miles MS, Funk SG, Carlson J. Parental Stressor Scale: neonatal intensive care unit. Nurs Res 1993;42:148-52.

31. Schmidt B, Roberts RS, Davis P, et al. Caffeine therapy for apnea of prematurity. N Engl J Med 2006;354:2112-21.

32. Piaggio G, Elbourne DR, Pocock SJ, et al. Reporting of noninferiority and equivalence randomized trials: extension of the CONSORT 2010 statement. JAMA 2012;308:2594-604.

33. Harris PA, Taylor R, Thielke R, et al. Research electronic data capture (REDCap) - a metadata-driven methodology and workflow process for providing translational research informatics support. $J$ Biomed Inform 2009;42:377-81. 